PROCEEDINGS OF THE

AMERICAN MATHEMATICAL SOCIETY

Volume 126, Number 1, January 1998, Pages 15-23

S 0002-9939(98)03911-2

\title{
THE CLASSIFICATION OF COMPLETE LIE ALGEBRAS WITH COMMUTATIVE NILPOTENT RADICAL
}

\author{
JIANG CUIPO AND MENG DAOJI
}

(Communicated by Roe Goodman)

\begin{abstract}
The work in this paper is a continuation of an earlier paper of the second author (Acta Math. 34 (1991), 191-202). We discuss the properties of finite-dimensional complete Lie algebras with abelian nilpotent radical over the complex field $\mathbf{C}$. We solve the problems of isomorphism, classification and realization of complete Lie algebras with commutative nilpotent radical.
\end{abstract}

\section{INTRODUCTION}

A Lie algebra $\mathcal{L}$ is called a complete Lie algebra if its centre $C(\mathcal{L})$ is zero and its derivations are all inner. The definition of complete Lie algebra was given by N. Jacobson in 1962 (cf. [8]). But the first important result - the derivation tower theorem - was obtained by E. V. Schenkman in 1951 (cf. [9]). In recent years, the theory of complete Lie algebras has been developing (see [1]-[7]). In [1], the properties of complete Lie algebras with commutative nilpotent radical have been discussed. The work in this paper is a continuation of [1].

Let $\mathcal{L}$ be a finite-dimensional Lie algebra over a field of characteristic zero. Then $\mathcal{L}$ has the Levi decomposition:

$$
\mathcal{L}=\mathfrak{s} \dot{+} \mathfrak{r}
$$

where $\mathfrak{s}$ is a maximal semisimple subalgebra of $\mathcal{L}$ and is called the Levi subalgebra of $\mathcal{L}$, and $\mathfrak{r}$ is the maximal solvable ideal of $\mathcal{L}$ and is called the radical of $\mathcal{L}$. The ideal

$$
\mathfrak{n}_{0}=[\mathcal{L}, \mathcal{L}] \cap \mathfrak{r}=[\mathcal{L}, \mathfrak{r}]
$$

is called the nilpotent radical of $\mathcal{L}$.

Since $[\mathfrak{s}, \mathfrak{r}] \subseteq \mathfrak{r}, \mathfrak{r}$ can be viewed as an $\mathfrak{s}$-module. The fact that $\mathfrak{s}$ is semisimple implies that $\mathfrak{r}$ can be decomposed into a direct sum of irreducible submodules. Let $\mathfrak{r}_{0}$ be the direct sum of trivial submodules, and $\mathfrak{r}_{n}$ the direct sum of non-trivial irreducible submodules. Denote by $C\left(\mathfrak{r}_{0}\right)$ the centre of $\mathfrak{r}_{0}$ and let $C_{\mathfrak{r}_{0}}\left(\mathfrak{r}_{n}\right)=\{x \in$ $\left.\mathfrak{r}_{0} \mid\left[x, \mathfrak{r}_{n}\right]=0\right\}$. It has been proved in [1] that $\mathcal{L}$ can be decomposed into the direct sum of complete ideals as follows:

$$
\mathcal{L}=\left(\mathfrak{s}+C\left(\mathfrak{r}_{0}\right)+\mathfrak{r}_{n}\right) \oplus C_{\mathfrak{r}_{0}}\left(\mathfrak{r}_{n}\right)
$$

Received by the editors July 6, 1995 and, in revised form, April 9, 1996.

1991 Mathematics Subject Classification. Primary 17B10, 17B20, 17B65, 17B67, 17 B68.

This research was supported in part by the National Science Foundation of China.

(C)1998 American Mathematical Society 
and $C_{\mathfrak{r}_{0}}\left(\mathfrak{r}_{n}\right)$ is an extension of abelian Lie algebra by abelian Lie algebra, and if the base field of $\mathcal{L}$ is algebraically closed, then $C_{\mathfrak{r}_{0}}\left(\mathfrak{r}_{n}\right)$ is a direct sum of 2-dimensional simple complete ideals. A complete Lie algebra is called a simple complete Lie algebra if it has no non-trivial complete ideals. By (1.3), if we study complete Lie algebras with commutative nilpotent radical, it is sufficient to discuss $\mathfrak{s}+C\left(\mathfrak{r}_{0}\right)+\mathfrak{r}_{n}$. In this case, $\mathfrak{n}_{0}=\mathfrak{r}_{n}$.

In [1], a complete Lie algebra $G=\mathfrak{g} \dot{+} V \dot{+} \mathfrak{a}$ over the complex field $\mathbf{C}$ was constructed in the following way. Let $\mathfrak{g}$ be a complex semisimple Lie algebra and $(\rho, V)$ a representation of $\mathfrak{g}$ which is decomposed into

$$
V=V_{1} \dot{+} V_{2} \dot{+} \cdots \dot{+} V_{n},
$$

where $V_{i}(i=1,2, \ldots, n)$ are irreducible invariant subspaces of $\rho$. Let $I_{i}(i=$ $1,2, \ldots, n)$ be the linear transformations of $V$ such that

$$
I_{i}\left(\sum_{j \neq i} V_{j}\right)=0,\left.\quad I_{i}\right|_{V_{i}}=\left.\mathrm{id}\right|_{V_{i}} .
$$

Let $\mathfrak{a}$ be the subalgebra of $\operatorname{gl}(V)$ generated by $I_{1}, I_{2}, \ldots, I_{n}$. Then $\mathfrak{a}$ is an abelian Lie algebra. Set

$$
G=\mathfrak{g} \dot{+} V \dot{+} \mathfrak{a} .
$$

The bracket in $G$ is defined by

$$
\left[x_{1}+v_{1}+A_{1}, x_{2}+v_{2}+A_{2}\right]=\left[x_{1}, x_{2}\right]+\rho\left(x_{1}\right) v_{2}-\rho\left(x_{2}\right) v_{1}-A_{2}\left(v_{1}\right)+A_{1}\left(v_{2}\right),
$$

where $x_{1}, x_{2} \in \mathfrak{g}, v_{1}, v_{2} \in V, A_{1}, A_{2} \in \mathfrak{a}$. Then $G$ is a complete Lie algebra with commutative nilpotent radical.

In this paper, we discuss the properties of finite-dimensional complete Lie algebras with commutative nilpotent radical $\mathfrak{n}_{0}=\mathfrak{r}_{n}$ over the complex field $\mathbf{C}$. We deduce that if $\mathfrak{r}_{n}$ is the direct sum of $t$ irreducible submodules, then the dimension of $\mathfrak{r}_{0}$ is $t$. We prove that $\mathfrak{r}_{n}$ can be decomposed properly so that the action of every element of $\operatorname{ad}_{\mathfrak{r}_{n}} \mathfrak{r}_{0}$ on each irreducible submodule is scalar. Therefore, finitedimensional complete Lie algebras with commutative nilpotent radical $\mathfrak{n}_{0}=\mathfrak{r}_{n}$ over $\mathbf{C}$ are in fact the Lie algebras constructed above. Hence, all finite-dimensional complete Lie algebras with commutative nilpotent radical over $\mathbf{C}$ are known.

\section{Some Lemmas}

Let

$$
\mathcal{L}=\mathfrak{s}+\mathfrak{r}=\mathfrak{s}+\left(\mathfrak{r}_{0}+\mathfrak{r}_{n}\right)
$$

be the Levi decomposition of $\mathcal{L}$. Then we have the following results.

Lemma 2.1 ([1]).

$$
\begin{aligned}
& {\left[\mathfrak{s}, \mathfrak{r}_{0}\right]=(0),} \\
& {\left[\mathfrak{s}, \mathfrak{r}_{n}\right]=\mathfrak{r}_{n},} \\
& {\left[\mathfrak{r}_{0}, \mathfrak{r}_{0}\right] \subseteq \mathfrak{r}_{0}}
\end{aligned}
$$

\section{Lemma 2.2.}

$$
\left[\mathfrak{r}_{0}, \mathfrak{r}_{n}\right] \subseteq \mathfrak{r}_{n}
$$


Proof. By (2.3) and (2.2), we have

$$
\begin{aligned}
{\left[\mathfrak{r}_{0}, \mathfrak{r}_{n}\right] } & =\left[\mathfrak{r}_{0},\left[\mathfrak{s}, \mathfrak{r}_{n}\right]\right] \subseteq\left[\left[\mathfrak{r}_{0}, \mathfrak{s}\right], \mathfrak{r}_{n}\right]+\left[\left[\mathfrak{r}_{n}, \mathfrak{r}_{0}\right], \mathfrak{s}\right] \\
& =\left[\mathfrak{s},\left[\mathfrak{r}_{0}, \mathfrak{r}_{n}\right]\right] \subseteq[\mathfrak{s}, \mathfrak{r}]=\left[\mathfrak{s}, \mathfrak{r}_{0}+\mathfrak{r}_{n}\right]=\mathfrak{r}_{n}
\end{aligned}
$$

The lemma holds.

Lemma 2.3. Let $\mathcal{L}$ be a Lie algebra with abelian nilpotent radical $\mathfrak{n}_{0}=\mathfrak{r}_{n}$. Then

$$
\left[\mathfrak{r}_{0}, \mathfrak{r}_{0}\right]=\left[\mathfrak{r}_{n}, \mathfrak{r}_{n}\right]=(0) .
$$

Proof. Since $\mathfrak{n}_{0}$ is commutative and $\mathfrak{n}_{0}=\mathfrak{r}_{n}$, we have

$$
\mathfrak{n}_{0}^{(1)}=\mathfrak{r}_{n}^{(1)}=\left[\mathfrak{r}_{n}, \mathfrak{r}_{n}\right]=(0)
$$

and

$$
\mathfrak{n}_{0}=\left[\mathfrak{s}+\mathfrak{r}_{0}+\mathfrak{r}_{n}, \mathfrak{r}_{0}+\mathfrak{r}_{n}\right]=\mathfrak{r}_{n}+\left[\mathfrak{r}_{0}, \mathfrak{r}_{0}\right] .
$$

The lemma follows from (2.4) and Lemma 2.2.

Let $\mathfrak{a}$ be an irreducible $\mathfrak{s}$-module. Then $\mathfrak{a}$ is a highest weight $\mathfrak{s}$-module since $\mathfrak{s}$ is semisimple and $\mathfrak{a}$ is finite dimensional.

Let

$$
\mathfrak{r}_{n}=\mathfrak{a}_{1} \oplus \mathfrak{a}_{2} \oplus \cdots \oplus \mathfrak{a}_{m}
$$

be the direct sum of submodules such that

$$
\mathfrak{a}_{i}=\mathfrak{a}_{i 1} \oplus \mathfrak{a}_{i 2} \oplus \cdots \oplus \mathfrak{a}_{i n_{i}} \quad(i=1,2, \ldots, m),
$$

where $\mathfrak{a}_{i k}\left(k=1,2, \ldots, n_{i}\right)$ are irreducible highest weight $\mathfrak{s}$-modules with highest weight $\lambda_{i}$ and, if $i \neq j$, then $\lambda_{i} \neq \lambda_{j}, i, j=1,2, \ldots, m$.

Denote by $z_{i j}\left(j=1,2, \ldots, n_{i}\right)$ the highest weight vectors of $\mathfrak{a}_{i j}\left(j=1,2, \ldots, n_{i}\right)$ respectively, and by $V_{i}$ the linear space with basis $\left\{z_{i 1}, \ldots, z_{i n_{i}}\right\}(i=1,2, \ldots, m)$. Then

$$
\operatorname{dim} V_{i}=n_{i}, \quad i=1,2, \ldots, m .
$$

Let $\mathfrak{h}_{0}$ be a Cartan subalgebra of $\mathfrak{s}$ and $\Delta_{0}$ the root system. Let $\Pi=\left\{\alpha_{1}, \alpha_{2}, \ldots\right.$, $\left.\alpha_{n}\right\}$ be the simple root system and $\mathfrak{s}=\mathfrak{h}_{0}+\sum_{\alpha \in \Delta_{0}} \mathfrak{s}_{\alpha}$ the root space decomposition with respect to $\mathfrak{h}_{0}$.

Lemma 2.4. Let $\mathcal{L}$ be a Lie algebra with commutative nilpotent radical, $D \in$ $\operatorname{Der}\left(\mathfrak{s}+\mathfrak{r}_{n}\right)$ be such that $D(\mathfrak{s})=(0)$ and $D\left(\mathfrak{r}_{n}\right) \subseteq \mathfrak{r}_{n}$. Then

$$
D\left(V_{i}\right) \subseteq V_{i}, \quad i=1,2, \ldots, m,
$$

and $D$ is uniquely determined by $\left.D\right|_{V_{i}}(i=1,2, \ldots, m)$.

Proof. Since $D \in \operatorname{Der}\left(\mathfrak{s}+\mathfrak{r}_{n}\right)$ and $D(\mathfrak{s})=0$, for any $h \in \mathfrak{h}_{0}$ we have

$$
D\left[h, z_{i j}\right]=\lambda_{i}(h) D z_{i j}
$$

Note that $z_{i j}$ is a highest weight vector of $\mathfrak{a}_{i j}$, therefore for $\alpha \in \Delta_{0}^{+}$and $e_{\alpha} \in \mathfrak{s}_{\alpha}$, we have

$$
\left[e_{\alpha}, z_{i j}\right]=0
$$

Therefore

$$
\left[e_{\alpha}, D z_{i j}\right]=D\left[e_{\alpha}, z_{i j}\right]=0
$$


It is clear from $(2.11)$ and $(2.12)$ that $D z_{i 1}, D z_{i 2}, \ldots, D z_{i n_{i}}(i=1,2, \ldots, m)$ are highest weight vectors associated to highest weight $\lambda_{i}(i=1,2, \ldots, m)$. So $D z_{i j} \in$ $V_{i}\left(j=1,2, \ldots, n_{i}, \quad i=1,2, \ldots, m\right)$. On the other hand, for $z \in \mathfrak{a}_{i j}, z$ has the form:

$$
z=\left[x_{1},\left[x_{2},\left[\ldots,\left[x_{q}, z_{i j}\right]\right] \ldots\right]\right]
$$

where $x_{i} \in \mathfrak{s}(i=1,2, \ldots, q)$. So

$$
D z=\left[x_{1},\left[x_{2},\left[\ldots,\left[x_{q}, D z_{i j}\right]\right] \ldots\right]\right] .
$$

The lemma is proved.

Lemma 2.5. Let $\mathcal{L}$ be a Lie algebra with abelian nilpotent radical $\mathfrak{n}_{0}=\mathfrak{r}_{n} . D \in$ $\operatorname{Der}\left(\mathfrak{s}+\mathfrak{r}_{n}\right)$ is such that $D(\mathfrak{s})=(0)$ and $D\left(\mathfrak{r}_{n}\right) \subseteq \mathfrak{r}_{n}$. Then

$$
D\left(\mathfrak{a}_{i}\right) \subseteq \mathfrak{a}_{i}, \quad i=1,2, \ldots, m .
$$

2) Set

$$
\mathcal{L}_{i}=\left\{D \in \operatorname{Der}\left(\mathfrak{s}+\mathfrak{r}_{n}\right) \mid D(\mathfrak{s})=(0), D\left(\mathfrak{r}_{n}\right) \subseteq \mathfrak{r}_{n} \text { and }\left.D\right|_{\mathfrak{a}_{j}}=0 \text {, if } j \neq i\right\} .
$$

Then the Lie algebra $\mathcal{L}_{i}$ is isomorphic to the general linear Lie algebra $\operatorname{gl}\left(V_{i}\right)$ which consists of all linear transformations of $V_{i}, i=1,2, \ldots, m$.

Proof. 1) follows from Lemma 2.4. Define

$$
\varphi(D)=\left.D\right|_{V_{i}}, \quad \text { for } D \in \mathcal{L}_{i}
$$

Then $\varphi$ is a linear mapping from $\mathcal{L}_{i}$ to $\operatorname{gl}\left(V_{i}\right)$. For $D_{1}, D_{2} \in \mathcal{L}_{i}$, if $D_{1} \neq D_{2}$, then from Lemma 2.4 we know $\varphi\left(D_{1}\right) \neq \varphi\left(D_{2}\right)$. Let $A \in \operatorname{gl}\left(V_{i}\right)$. Define the linear transformation $D$ of $\mathfrak{s}+\mathfrak{r}_{n}$ by

$$
\begin{gathered}
D(\mathfrak{s})=0,\left.D\right|_{\mathfrak{a}_{j}}=0, \quad \text { if } j \neq i, \\
D\left[x_{1},\left[x_{2}, \ldots,\left[x_{q}, z_{i j}\right]\right] \ldots\right]=\left[x_{1},\left[x_{2}, \ldots,\left[x_{q}, A z_{i j}\right]\right] \ldots\right] \quad\left(j=1,2, \ldots, n_{i}\right),
\end{gathered}
$$

where $x_{1}, x_{2}, \ldots, x_{q} \in \mathfrak{s}$. Then $D \in \operatorname{Der}\left(\mathfrak{s}+\mathfrak{r}_{n}\right)$. So $\varphi$ is a bijection.

For $D_{1}, D_{2} \in \mathcal{L}_{i}$, we have

$$
\begin{aligned}
\varphi\left[D_{1}, D_{2}\right] & =\left.\left[D_{1}, D_{2}\right]\right|_{V_{i}} \\
& =\left.D_{1} D_{2}\right|_{V_{i}}-\left.D_{2} D_{1}\right|_{V_{i}}=\left.\left.D_{1}\right|_{V_{i}} D_{2}\right|_{V_{i}}-\left.\left.D_{2}\right|_{V_{i}} D_{1}\right|_{V_{i}} \\
& =\left[\varphi\left(D_{1}\right), \varphi\left(D_{2}\right)\right] .
\end{aligned}
$$

Hence $\varphi$ is a homomorphism from the Lie algebra $\mathcal{L}_{i}$ to the Lie algebra gl $\left(V_{i}\right)$. The lemma holds.

\section{THE STRUCTURE OF RADICAL $\mathfrak{r}$}

Lemma 3.1. Let $D$ be an inner derivation of $\mathcal{L}$ and $D(\mathfrak{s})=(0)$. Then there exists an element $y \in \mathfrak{r}_{0}$ such that

$$
D=\operatorname{ad} y
$$

Proof. Since $D$ is an inner derivation of $\mathcal{L}$, there exist $x \in \mathfrak{s}, y \in \mathfrak{r}_{0}, z \in \mathfrak{r}_{n}$ such that

$$
D=\operatorname{ad}(x+y+z) .
$$

$D(\mathfrak{s})=(0)$ implies that

$$
[x+y+z, \mathfrak{s}]=[x, \mathfrak{s}]+[z, \mathfrak{s}]=(0) .
$$


From the fact that $[x, \mathfrak{s}] \subseteq \mathfrak{s},[z, \mathfrak{s}] \subseteq \mathfrak{r}_{n}$, we have

$$
[x, \mathfrak{s}]=(0), \quad[z, \mathfrak{s}]=(0) \text {. }
$$

But $\mathfrak{s}$ is semisimple and $\mathfrak{r}_{n}$ is the direct sum of non-trivial submodules. Therefore

$$
x=z=0 .
$$

Lemma 3.2. Let $\mathcal{L}$ be a Lie algebra with trivial centre and commutative nilpotent radical $\mathfrak{n}_{0}=\mathfrak{r}_{n}$. Then

1) $\mathfrak{r}_{0}$ is isomorphic to $\operatorname{ad}_{\mathfrak{r}_{n}} \mathfrak{r}_{0}$.

2) For $x \in \mathfrak{r}_{0}$, we have

$$
\operatorname{ad}_{\mathfrak{r}_{n}} x\left(\mathfrak{a}_{i}\right) \subseteq \mathfrak{a}_{i},\left.\quad \operatorname{ad}_{\mathfrak{r}_{n}} x\right|_{V_{i}} \in \operatorname{gl}\left(V_{i}\right) \quad(i=1,2, \ldots, m) .
$$

Lemma 3.3. Let $\mathcal{L}$ be a complete Lie algebra with commutative nilpotent radical $\mathfrak{n}_{0}=\mathfrak{r}_{n}$. For $x \in \mathfrak{r}_{0}$, define the linear transformations $D_{i}(i=1,2, \ldots, m)$ of $\mathcal{L}$ by

$$
\left.D_{i}\right|_{\mathfrak{s}+\mathfrak{r}_{0}}=0,\left.\quad D_{i}\right|_{\mathfrak{a}_{i}}=\left.\operatorname{ad} x\right|_{\mathfrak{a}_{i}},\left.\quad D_{i}\right|_{\mathfrak{a}_{j}}=0 \quad(j=1, \ldots, i-1, i+1, \ldots, m) .
$$

Then there exist $y_{1}, y_{2}, \ldots, y_{m} \in \mathfrak{r}_{0}$ such that

$$
D_{i}=\operatorname{ad} y_{i} \quad(i=1,2, \ldots, m) .
$$

Proof. For $s_{1}, s_{2} \in \mathfrak{s}, x_{1}, x_{2} \in \mathfrak{r}_{0}, y_{1}, y_{2} \in \mathfrak{a}_{i}, z_{1}, z_{2} \in \mathfrak{a}_{1}+\cdots+\mathfrak{a}_{i-1}+\mathfrak{a}_{i+1}+\cdots+\mathfrak{a}_{m}$, by 2) of Lemma 3.2, we have $\left[x_{1}, y_{2}\right],\left[x_{2}, y_{1}\right] \in \mathfrak{a}_{i},\left[x_{1}, z_{2}\right],\left[x_{2}, z_{1}\right] \in \mathfrak{a}_{1}+\cdots+\mathfrak{a}_{i-1}+$ $\mathfrak{a}_{i+1}+\cdots+\mathfrak{a}_{m}$. So by $(2.2)$ and (2.6) we deduce that

$$
\begin{aligned}
D_{i} & {\left[s_{1}+x_{1}+y_{1}+z_{1}, s_{2}+x_{2}+y_{2}+z_{2}\right] } \\
= & \operatorname{ad} x\left(\left[s_{1}, y_{2}\right]+\left[x_{1}, y_{2}\right]+\left[y_{1}, s_{2}\right]+\left[y_{1}, x_{2}\right]\right) \\
= & {\left[s_{1}, \operatorname{ad} x\left(y_{2}\right)\right]+\left[x_{1}, \operatorname{ad} x\left(y_{2}\right)\right]+\left[\operatorname{ad} x\left(y_{1}\right), s_{2}\right]+\left[\operatorname{ad} x\left(y_{1}\right), x_{2}\right], } \\
{\left[D_{i}\left(s_{1}+x_{1}+y_{1}+z_{1}\right), s_{2}+x_{2}+y_{2}+z_{2}\right] } & \quad+\left[s_{1}+x_{1}+y_{1}+z_{1}, D_{i}\left(s_{2}+x_{2}+y_{2}+z_{2}\right)\right] \\
& \quad\left[\operatorname{ad} x\left(y_{1}\right), s_{2}+x_{2}+y_{2}+z_{2}\right]+\left[s_{1}+x_{1}+y_{1}+z_{1}, \operatorname{ad} x\left(y_{2}\right)\right] \\
= & {\left[\operatorname{ad} x\left(y_{1}\right), s_{2}\right]+\left[\operatorname{ad} x\left(y_{1}\right), x_{2}\right]+\left[s_{1}, \operatorname{ad} x\left(y_{2}\right)\right]+\left[x_{1}, \operatorname{ad} x\left(y_{2}\right)\right] . }
\end{aligned}
$$

Therefore $D_{i} \in \operatorname{Der} \mathcal{L}(i=1,2, \ldots, m)$. Since $\mathcal{L}$ is a complete Lie algebra, there exist $y_{1}, y_{2}, \ldots, y_{m} \in \mathcal{L}$ such that

$$
D_{i}=\operatorname{ad} y_{i} \quad(i=1,2, \ldots, m) .
$$

By Lemma 3.1, $y_{i} \in \mathfrak{r}_{0}(i=1,2, \ldots, m)$.

Theorem 3.1. Let $\mathcal{L}$ be a complete Lie algebra with commutative nilpotent radical $\mathfrak{n}_{0}=\mathfrak{r}_{n}$. Set

$$
\begin{array}{r}
\mathfrak{h}_{i}=\left\{\operatorname{ad} x \mid x \in \mathfrak{r}_{0} \text { and }\left.\operatorname{ad} x\right|_{\mathfrak{a}_{j}}=0(j=1, \ldots, i-1, i+1, \ldots, m)\right\} \\
i=1,2, \ldots, m,
\end{array}
$$

Then

1) $\left.\mathfrak{h}_{i}\right|_{\mathfrak{s}+\mathfrak{r}_{n}}$ is a commutative subalgebra of $\mathcal{L}_{i}$ and

$$
\operatorname{ad} \mathfrak{r}_{0}=\left.\left.\mathfrak{h}_{1} \oplus \mathfrak{h}_{2} \oplus \cdots \oplus \mathfrak{h}_{m} \simeq \mathfrak{h}_{1}\right|_{\mathfrak{s}+\mathfrak{r}_{n}} \oplus \cdots \oplus \mathfrak{h}_{m}\right|_{\mathfrak{s}+\mathfrak{r}_{n}}
$$

2) $\mathfrak{h}_{i}$ is isomorphic to $H_{i}$ which is an abelian subalgebra of $\operatorname{gl}\left(V_{i}\right), i=1,2, \ldots, m$. 
Proof. The proof follows from Lemma 3.3 and Lemma 2.5.

Lemma 3.4. Let $\mathcal{L}$ be a Lie algebra with commutative nilpotent radical $\mathfrak{n}_{0}=\mathfrak{r}_{n}$. Let $D \in \operatorname{Der}\left(\mathfrak{s}+\mathfrak{r}_{n}\right)$ be such that $D(\mathfrak{s})=0$ and $D\left(\mathfrak{r}_{n}\right) \subseteq \mathfrak{r}_{n}$. Extend $D$ to the linear transformation of $\mathcal{L}$ by

$$
\left.D\right|_{\mathfrak{r}_{0}}=0
$$

Then $D$ is a derivation of $\mathcal{L}$ if and only if

$$
\left[D, \operatorname{ad}_{\mathfrak{s}+\mathfrak{r}_{n}} \mathfrak{r}_{0}\right]=0 .
$$

Proof. $D \in$ Der $\mathcal{L}$ if and only if the formula

$$
\begin{aligned}
& D\left[x_{1}+y_{1}+z_{1}, x_{2}+y_{2}+z_{2}\right] \\
& \quad=\left[D\left(x_{1}+y_{1}+z_{1}\right), x_{2}+y_{2}+z_{2}\right]+\left[x_{1}+y_{1}+z_{1}, D\left(x_{2}+y_{2}+z_{2}\right)\right]
\end{aligned}
$$

holds, for any $x_{1}, x_{2} \in \mathfrak{s}, y_{1}, y_{2} \in \mathfrak{r}_{0}, z_{1}, z_{2} \in \mathfrak{r}_{n}$.

Note that

$$
\begin{aligned}
& D\left[x_{1}+y_{1}+z_{1}, x_{2}+y_{2}+z_{2}\right] \\
& \quad=D\left[z_{1}, x_{1}\right]+D\left[z_{1}, y_{2}\right]+D\left[x_{1}, z_{2}\right]+D\left[y_{1}, z_{2}\right]+D\left[z_{1}, z_{2}\right] \\
& \quad\left[D\left(x_{1}+y_{1}+z_{1}\right), x_{2}+y_{2}+z_{2}\right]+\left[x_{1}+y_{1}+z_{1}, D\left(x_{2}+y_{2}+z_{2}\right)\right] \\
& \quad=\left[D z_{1}, x_{2}\right]+\left[D z_{1}, y_{2}\right]+\left[x_{1}, D z_{2}\right]+\left[y_{1}, D z_{2}\right]+\left[D z_{1}, z_{2}\right]+\left[z_{1}, D z_{2}\right]
\end{aligned}
$$

and $D \in \operatorname{Der}\left(\mathfrak{s}+\mathfrak{r}_{n}\right)$ is such that $D(\mathfrak{s})=0$. Therefore $D \in \operatorname{Der} \mathcal{L}$ if and only if

$$
D[y, z]=[y, D z], \quad \text { for any } y \in \mathfrak{r}_{0}, z \in \mathfrak{r}_{n},
$$

i.e.,

$$
\left[D, \operatorname{ad}_{\mathfrak{s}+\mathfrak{r}_{n}} \mathfrak{r}_{0}\right]=0 .
$$

Theorem 3.2. Let $\mathcal{L}$ be a complete Lie algebra with abelian nilpotent radical $\mathfrak{n}_{0}=$ $\mathfrak{r}_{n}$, and let $\mathfrak{h}_{i}, H_{i}, \mathcal{L}_{i}(i=1,2, \ldots, m)$ be as above. Then

$$
\begin{gathered}
C_{\mathcal{L}_{i}}\left(\left.\mathfrak{h}_{i}\right|_{\mathfrak{s}+\mathfrak{r}_{n}}\right)=\left.\mathfrak{h}_{i}\right|_{\mathfrak{s}+\mathfrak{r}_{n}}, \quad i=1,2, \ldots, m, \\
C_{\mathrm{gl}\left(V_{i}\right)}\left(H_{i}\right)=H_{i}, \quad i=1,2, \ldots, m .
\end{gathered}
$$

Proof. Since $\mathfrak{r}_{0}$ is commutative, we have

$$
\left.\mathfrak{h}_{i}\right|_{\mathfrak{s}+\mathfrak{r}_{n}} \subseteq C_{\mathcal{L}_{i}}\left(\left.\mathfrak{h}_{i}\right|_{\mathfrak{s}+\mathfrak{r}_{n}}\right) .
$$

Let $D_{i} \in \mathcal{L}_{i}$ be such that $\left[D_{i},\left.\mathfrak{h}_{i}\right|_{\mathfrak{s}+\mathfrak{r}_{n}}\right]=0$. Then by (3.3) we have

$$
\left[D_{i}, \operatorname{ad}_{\mathfrak{s}+\mathfrak{r}_{n}} \mathfrak{r}_{0}\right]=0 .
$$

Extend $D_{i}$ to the linear transformation of $\mathcal{L}$ by

$$
\left.D_{i}\right|_{\mathfrak{r}_{0}}=0
$$

Then by Lemma 3.4, $D_{i}$ is a derivation of $\mathcal{L}$. Note that $\mathcal{L}$ is a complete Lie algebra, therefore

$$
D_{i}=\operatorname{ad} z_{i} \in \mathfrak{h}_{i}, \quad z_{i} \in \mathfrak{r}_{0} \quad(i=1,2, \ldots, m) .
$$

Hence $\left.\left.D_{i}\right|_{\mathfrak{s}+\mathfrak{r}_{n}} \in \mathfrak{h}_{i}\right|_{\mathfrak{s}+\mathfrak{r}_{n}}(i=1,2, \ldots, m)$. This proves (3.4). (3.5) follows from the fact that $\mathcal{L}_{i}$ is isomorphic to $\mathrm{gl}\left(V_{i}\right)$ and $\left.\mathfrak{h}_{i}\right|_{\mathfrak{s}+\mathfrak{r}_{n}}$ is isomorphic to $H_{i}$. 
Corollary 3.1. There exist elements ad $x_{i} \in \mathfrak{h}_{i}(i=1,2, \ldots, m)$ such that

$$
\left.\operatorname{ad} x_{i}\right|_{\mathfrak{a}_{i}}=\left.\mathrm{id}\right|_{\mathfrak{a}_{i}} \quad(i=1,2, \ldots, m) .
$$

Thus $H_{i}$ contains identical transformations $I_{i}=\left.\mathrm{id}\right|_{V_{i}}, i=1,2, \ldots, m$.

From Corollary 3.1, we have

$$
H_{i}=H_{i}^{\prime} \oplus \mathbf{C} I_{i}
$$

and $H_{i}^{\prime} \subseteq \operatorname{sl}\left(V_{i}\right)(i=1,2, \ldots, m)$, where $\operatorname{sl}\left(V_{i}\right)$ is the special linear Lie algebra on $V_{i}$. Since $\mathfrak{h}_{i}$ is isomorphic to $H_{i}$, we have

$$
\mathfrak{h}_{i}=\mathfrak{h}_{i}^{\prime} \oplus \mathbf{C} \text { ad } x_{i} \quad(i=1,2, \ldots, m),
$$

where $\mathfrak{h}_{i}^{\prime}$ is isomorphic to $H_{i}^{\prime}$ and $\operatorname{ad} x_{i}$ is the same as in Corollary 3.1. In fact, $\left.\mathfrak{h}_{i}^{\prime}\right|_{V_{i}}=H_{i}^{\prime}$, ad $\left.x_{i}\right|_{V_{i}}=I_{i}(i=1,2, \ldots, m)$.

We will show that $H_{i}^{\prime}$ is a maximal torus subalgebra of $\operatorname{sl}\left(V_{i}\right), i=1, \ldots, m$.

Lemma 3.5. Let $\mathcal{L}$ be a Lie algebra with trivial centre and nilpotent radical $\mathfrak{n}_{0}=$ $\mathfrak{r}_{n}$. Let $D_{1} \in \operatorname{Der}\left(\mathfrak{s}+\mathfrak{r}_{n}\right)$ be such that $D_{1}(\mathfrak{s})=(0)$ and $D_{1}\left(\mathfrak{r}_{n}\right) \subseteq \mathfrak{r}_{n}$, and

$$
\left[D_{1}, \operatorname{ad}_{\mathfrak{s}+\mathfrak{r}_{n}} \mathfrak{r}_{0}\right] \subseteq \operatorname{ad}_{\mathfrak{s}+\mathfrak{r}_{n}} \mathfrak{r}_{0} .
$$

Then there exists $D \in$ Der $\mathcal{L}$ such that

$$
\left.D\right|_{\mathfrak{s}+\mathfrak{r}_{n}}=D_{1}
$$

and if $\left[D_{1}, \operatorname{ad}_{\mathfrak{s}+\mathfrak{r}_{n}} \mathfrak{r}_{0}\right] \neq 0$, then $D$ is an outer derivation of $\mathcal{L}$.

Proof. From (3.8), for any $x \in \mathfrak{r}_{0}$ there exists $y \in \mathfrak{r}_{0}$ such that

$$
\left[D_{1}, \operatorname{ad}_{\mathfrak{s}+\mathfrak{r}_{n}} x\right]=\operatorname{ad}_{\mathfrak{s}+\mathfrak{r}_{n}} y .
$$

Define the transformation $D_{2}$ of $\mathfrak{r}_{0}$ by

$$
D_{2}(x)=y \text {. }
$$

By Lemma 3.2 there is no ambiguity in the definition of $D_{2}$. It is clear that $D_{2}$ is a linear transformation of $\mathfrak{r}_{0} . D_{2}$ is a derivation of $\mathfrak{r}_{0}$ since $\mathfrak{r}_{0}$ is commutative. From (3.8), for $x \in \mathfrak{r}_{0}$ and $z \in \mathfrak{r}_{n}$ we have

$$
D_{1}[x, z]=\left[x, D_{1} z\right]+\left[D_{2} x, z\right] .
$$

Define the linear transformation $D$ of $\mathcal{L}$ by

$$
\left.D\right|_{\mathfrak{s}+\mathfrak{r}_{n}}=D_{1},\left.\quad D\right|_{\mathfrak{r}_{0}}=D_{2} .
$$

Then for any $x_{1}, x_{2} \in \mathfrak{s}, y_{1}, y_{2} \in \mathfrak{r}_{0}, z_{1}, z_{2} \in \mathfrak{r}_{n}$, we have

$$
\begin{aligned}
& D\left[x_{1}+y_{1}+z_{1}, x_{2}+y_{2}+z_{2}\right] \\
& =\left[D_{1} z_{1}, x_{2}\right]+D_{1}\left[z_{1}, y_{2}\right]+\left[x_{1}, D_{1} z_{2}\right]+D_{1}\left[y_{1}, z_{2}\right]+D_{1}\left[z_{1}, z_{2}\right], \\
& \quad\left[D\left(x_{1}+y_{1}+z_{1}\right), x_{2}+y_{2}+z_{2}\right]+\left[x_{1}+y_{1}+z_{1}, D\left(x_{2}+y_{2}+z_{2}\right)\right] \\
& \quad=\left[D_{1} z_{1}, x_{2}\right]+\left[D_{1} z_{1}, y_{2}\right]+\left[x_{1}, D_{1} z_{2}\right]+\left[y_{1}, D_{1} z_{2}\right]+\left[D_{1} z_{1}, z_{2}\right] \\
& \quad+\left[z_{1}, D_{1} z_{2}\right]+\left[D_{2} y_{1}, z_{2}\right]+\left[z_{1}, D_{2} y_{2}\right] .
\end{aligned}
$$

Since $D_{1} \in \operatorname{Der}\left(\mathfrak{s}+\mathfrak{r}_{n}\right)$ is such that $D_{1}(\mathfrak{s})=(0)$ and (3.9) holds, we have

$$
\begin{aligned}
& D\left[x_{1}+y_{1}+z_{1}, x_{2}+y_{2}+z_{2}\right] \\
& \quad=\left[D\left(x_{1}+y_{1}+z_{1}\right), x_{2}+y_{2}+z_{2}\right]+\left[x_{1}+y_{1}+z_{1}, D\left(x_{2}+y_{2}+z_{2}\right)\right] .
\end{aligned}
$$

Hence $D \in \operatorname{Der} \mathcal{L}$. If $D \in \operatorname{ad} \mathcal{L}$, then by Lemma 3.1, we have

$$
D_{1} \in \operatorname{ad}_{\mathfrak{s}+\mathfrak{r}_{n}} \mathfrak{r}_{0} .
$$


But $\mathfrak{r}_{0}$ is commutative. Therefore,

$$
\left[D_{1}, \operatorname{ad}_{\mathfrak{s}+\mathfrak{r}_{n}} \mathfrak{r}_{0}\right]=0
$$

Thus we have completed the proof.

Theorem 3.3. Let $\mathcal{L}$ be a complete Lie algebra with commutative nilpotent radical $\mathfrak{n}_{0}=\mathfrak{r}_{n}$. Then $H_{i}^{\prime}$ is a maximal torus subalgebra of $\operatorname{sl}\left(V_{i}\right), i=1,2, \ldots, m$.

Proof. Let $T_{i} \in \operatorname{sl}\left(V_{i}\right)$ be such that $\left[T_{i}, H_{i}\right] \subseteq H_{i}$. Then $D_{i}=\varphi^{-1}\left(T_{i}\right) \in \mathcal{L}_{i}$, and $D_{i}$ satisfies the conditions of Lemma 3.5. Thus there exists $D_{i}^{\prime} \in \operatorname{Der} \mathcal{L}$ such that

$$
\left.D_{i}^{\prime}\right|_{\mathfrak{s}+\mathfrak{r}_{n}}=D_{i}, \quad i=1,2, \ldots, m .
$$

Note that $\mathcal{L}$ is a complete Lie algebra, so by Lemma 3.1, we have

$$
D_{i}^{\prime} \in \operatorname{ad}_{\mathcal{L}} \mathfrak{r}_{0}, \quad i=1,2, \ldots, m .
$$

The fact that $\left.D_{i}\right|_{\mathfrak{a}_{j}}=0$ (when $\left.j \neq i\right)$ implies $D_{i} \in \mathfrak{h}_{i}(i=1,2, \ldots, m)$. Thus we have

$$
T_{i}=\varphi\left(\left.D_{i}^{\prime}\right|_{\mathfrak{s}+\mathfrak{r}_{n}}\right)=\varphi\left(D_{i}\right) \in H_{i}^{\prime}, \quad i=1,2, \ldots, m .
$$

From this we know that $H_{i}^{\prime}$ is a self-normal subalgebra of $\operatorname{sl}\left(V_{i}\right)$. Since $H_{i}^{\prime}$ is commutative, we deduce that $H_{i}^{\prime}$ is a Cartan subalgebra of $\operatorname{sl}\left(V_{i}\right)$. $\operatorname{But} \operatorname{sl}\left(V_{i}\right)$ is a simple Lie algebra, therefore $H_{i}^{\prime}$ is a maximal torus subalgebra of $\operatorname{sl}\left(V_{i}\right)$.

Corollary 3.2. Let $\mathcal{L}$ be a complete Lie algebra with abelian nilpotent radical $\mathfrak{n}_{0}=$ $\mathfrak{r}_{n}$. Then $H_{i}=H_{i}^{\prime}+\mathbf{C} I_{i}$ is a maximal torus subalgebra of $\operatorname{gl}\left(V_{i}\right), i=1,2, \ldots, m$.

Corollary 3.3. Let $\mathcal{L}$ be a complete Lie algebra with abelian nilpotent radical $\mathfrak{n}_{0}=$ $\mathfrak{r}_{n}$. Then

$$
\operatorname{dim} \mathfrak{h}_{i}=n_{i}, \quad i=1,2, \ldots, m
$$

Thus we have

$$
\operatorname{dim} \mathfrak{r}_{0}=n_{1}+n_{2}+\cdots+n_{m} .
$$

Since $H_{i}$ is a maximal torus subalgebra of $\mathrm{gl}\left(V_{i}\right)$, we can choose a basis $\left\{y_{i 1}\right.$, $\left.y_{i 2}, \ldots, y_{i n_{i}}\right\}$ of $V_{i}$ such that the matrix of every element of $H_{i}$ relative to the basis is diagonal, $i=1,2, \ldots, m$. On the other hand, $y_{i 1}, y_{i 2}, \ldots, y_{i n_{i}}$ are highest weight vectors associated to highest weight $\lambda_{i}$. So the highest weight $\mathfrak{s}$-module $\mathfrak{a}_{i j}^{\prime}$ whose highest weight vector is $y_{i j}$ is irreducible and

$$
\mathfrak{a}_{i}=\mathfrak{a}_{i 1}^{\prime} \oplus \mathfrak{a}_{i 2}^{\prime} \oplus \cdots \oplus \mathfrak{a}_{i n_{i}}^{\prime}, \quad i=1,2, \ldots, m .
$$

From this we deduce that

Theorem 3.4. Let $\mathcal{L}$ be a complete Lie algebra with abelian nilpotent radical $\mathfrak{n}_{0}=$ $\mathfrak{r}_{n}$. Let $\mathfrak{r}_{n}$ be the direct sum of $t$ irreducible submodules. Then

$$
\operatorname{dim} \mathfrak{r}_{0}=t
$$

and $\mathfrak{r}_{n}$ can be decomposed properly into the direct sum of irreducible submodules:

$$
\mathfrak{r}_{n}=\mathfrak{m}_{1} \oplus \mathfrak{m}_{2} \oplus \cdots \oplus \mathfrak{m}_{t}
$$

so that

$$
\mathfrak{r}_{0} \simeq \operatorname{ad}_{\mathfrak{r}_{n}} \mathfrak{r}_{0}=\mathbf{C} I_{1} \oplus \mathbf{C} I_{2} \oplus \cdots \oplus \mathbf{C} I_{t}
$$


where $I_{i}$ is the linear transformation of $\mathfrak{r}_{n}$ such that

$$
I_{i}\left(\sum_{j \neq i} \mathfrak{m}_{j}\right)=(0),\left.\quad I_{i}\right|_{\mathfrak{m}_{i}}=\left.\mathrm{id}\right|_{\mathfrak{m}_{i}} \quad(i=1,2, \ldots, t) .
$$

So $\mathcal{L}$ is in fact the complete Lie algebra constructed in section 1 .

Theorem 3.5. Let $\mathcal{L}_{1}$ and $\mathcal{L}_{2}$ be complete Lie algebras. Let $\mathfrak{n}_{i}$ be the nilpotent radical of $\mathcal{L}_{i}$ and $\mathfrak{s}_{i}$ be the Levi subalgebra of $\mathcal{L}_{i}(i=1,2)$. Then $\mathcal{L}_{1}$ is isomorphic to $\mathcal{L}_{2}$ if and only if the Lie algebra $\mathfrak{s}_{1}$ is isomorphic to $\mathfrak{s}_{2}$ and the $\mathfrak{s}_{1}$-module $\mathfrak{n}_{1}$ is isomorphic to the $\mathfrak{s}_{2}$-module $\mathfrak{n}_{2}$.

Theorem 3.6. Let $\mathfrak{s}$ be a semisimple Lie algebra and $\mathfrak{n}$ an $\mathfrak{s}$-module. Define

$$
\left[s_{1}+x_{1}, s_{2}+x_{2}\right]=\left[s_{1}, s_{2}\right]+s_{1}\left(x_{2}\right)-s_{2}\left(x_{1}\right)
$$

where $s_{1}, s_{2} \in \mathfrak{s}, x_{1}, x_{2} \in \mathfrak{n}$. Then there is a unique up to isomorphism complete Lie algebra $\mathcal{L}$ such that $\mathfrak{n}$ is its nilpotent radical and $\mathfrak{s}$ is its Levi subalgebra and its bracket satisfies (3.12).

\section{REFERENCES}

1. D. J. Meng, The complete Lie algebras with abelian nilpotent radical, Acta. Math. 34 (1991), 191-202. (Chinese) MR 92f:17004

2. _ On complete Lie algebras, Acta. Sci. Nat. Univ. Nankai 2 (1985), 9-10. (Chinese)

3. - The uniqueness of the decomposition of complete Lie algebras, Acta. Sci. Nat. Univ. Nankai 3 (1990), 23-26. (Chinese)

4. 5507. MR 95h:17006

5. - Complete Lie algebras and Heisenberg algebras, Communications in Algebra 22 (1994), 5509-5524. MR 95j:17031

6. D. J. Meng and S. P. Wang, On the construction of complete Lie algebras, Journal of Algebra 176 (1995), 621-637. MR 96h:17007

7. E. L. Stitzinger, On Lie algebras with only inner derivations, J. of Algebra 105 (1987), 341-343. MR 88k:17003

8. N. Jacobson, Lie algebras, Wiley (Interscience), New York, 1962. MR 26:1345

9. E. V. Schenkman, A theory of subinvariant Lie algebras, Amer. J. Math. 73 (1951), 453-474. MR 13:103a

Department of Mathematics, Yantai Teachers University, Yantai 264025, China

Department of Mathematics, Nankai University, Tianjin 300071, China 